\title{
ANNUAL INDEX 1993
}

\section{AUTHOR INDEX}

Adams, A. T., see Halvorson Aker, P. D., see Kirkaldy-Willis

Biewen, P. and Taylor, T., Resistance Training for Rehabilitation of Disability Secondary to LBP, 3(4):32-35

Billings, J., see Boland

Blunk, K. L., see Gudas

Boland, P. J., Billings, J., Healey, J. H., The Management of Pathologic Fractures, 3(2):27-34

Bookhout, M. R., Evaluation and Conservátive Management of Spondylolisthesis, 3(4):24-31

Brena, S. F. and Spektor, S., Systematic Assessment of Impairment and Residual Functional Capacity in Pain-Impaired Patients, 3(1):6-19

Brennan, M. J. and Warfel, B. S., Musculoskeletal Complications of Cancer: A Survey of Fifty Patients 3(2): $1-6$

Brennan, M. J., Overview of Physiatric Approaches to Cancer Pain, 3(2):53-59

Bunger, S. D., see Trinkle

Burns, S. H., see Kirkaldy-Willis

Buschbacher, R., The Use and Abuse of Ankle Supports in Sports Injuries, 3(3):57-68

Carruth, M., ???FCE-WTS-WH-WCEFFD-WC???, 3(4):86-94

Cherny, N. I. and Portenoy, R. K., Pharmacotherapy of Cancer Pain: A Practical Guide, 3(2):7-26

Cocchiarella, A., see Singer

Decker, M. J., Point/Counterpoint: A Response to Redefining Chronic Pain Programs for the Injured Worker Population, 3(1):88-90

Delgado, S. and Runke, J. A., Pro: Impairment Listing for Pain in Social Security Disability Determination, 3(1):64-70

Deyo, R. A., Clinical Strategies for Controlling Costs and Improving Quality in the Primary Care of LBP, 3(4):1-13

Dotson, L., see Fiebert
Fiebert, M., Peterman, D., Dotson, L., Thoracic Segmental Flexion During Cervical Forward Bending, 3(4):80-85

Fiebert, M. and Spyropoulos, T., Overview of Functional Rehabilitation of Low Back Patients, 3(4):36-49

Fortin, J. D., Sacroiliac Joint Dysfunction, 3(3):31-43

Goldish, G., Introduction: Lumbar Spinal Orthotics, 3(3):1-11

Gudas, S. A. and Blunk, K. L. Cancer Rehabilitation Approaches to Neurologic Pain Syndromes in Malignancy, 3(4):68-79

Halvorson, K., Adams, A. 'T., Halvorson, G. A., Shidawara, C., A Program of Functional Progression for Low Back Patients, 3(4):50-60

Halvorson, G. A., see Halvorson $\mathrm{K}$

Harden, R. N., The Neurophysiology of Pain: A Clinical Summary, $3(1): 1-5$

Hart, D. L., see Trinkle

Healey, J. H., see Boland

Johnson, E. W., Communication and Medical Devices: A Clarification, 3(3):ed.

Johnson, E. W., Low Back Pain, 3(4): ed.

Kim, S. S., Spondylolisthesis, 3(4):14-22

Kirkaldy-Willis, W. H., Aker, P. D., Burns, S. H., Elastic Supports and Body Suits: Effective EnergyStoring Garments, 3(3):12-20

Kreider, J. R., Case Management: A Clinical or Sales Function?, 3(1):76-82

Kreider, J. R., 'The Need for Redefining Chronic Pain Programs for the Injured Worker Population, 3(1):83-87

Krol, G., Evaluation of Neoplastic Involvement of Brachial and Lumbosacral Plexus 3(2):35-43

LaBan, M. M., Physiatric Management of Patients with Metastatic Disease of the Spine, 3(2):69-77

Lachmann, E. S., see Warfel

Long, S. P. and Rafii, A., The Multidisciplinary Management of the Chronic Pain Patient, $3(1): 48-53$
McDonnell, M. E. and Shea, B. D., The Role of Physical Therapy in Patients with Metastatic Disease to Bone, 3(2):78-84

Merskey, H., Con: Impairment Listing for Pain in Social Security Disability Determination, 3(1):71-75

Mueller, M., see Tanner

Nagler, W., see Warfel

Ostermann, H., see Tanner

Peay, A., see Wells

Peterman, D., see Fiebert

Portenoy, R. K., Evaluation of Back Pain Caused by Epidural Neoplasm, 3(2):44-52

Portenoy, R. K., see Cherney

Rafii, A., see Long

Runke, J. A., see Delgado

Saunders, H. D., Regarding the Controversy of Lumbosacral Supports and Braces-An Update, 3(3):21-30

Shea, B. D., see McDonnell

Shidawara, C., see Halvorson G. A

Singer, G. L. and Cocchiarella, A., Juxtafacet Cysts as a Cause of Low Back Pain, 3(3):69-73

Spektor, S., see Brena

Spyropoulos, T., see Fiebert

Tait, R. C., Psychological Factors in the Assessment of Disability among Patients with Chronic Pain, 3(1):20-47

Tanner, R., Mueller M., Ostermann, H., Orthotic Management of Osteoporosis, 3(3):44-56

Taylor, T., see Biewen

Trinkle, K. L., Bunger, S. D., Hart, D. L., Roles and Responsibilities of Team Members in the FCE and Rehabilitation of the Injured Worker, 3(4)61-67

Warfel, B. S., Lachmann, E. S., Nagler, W., Physiatric Evaluation of the Cancer Patient, 3(2):60-68

Warfel, B. S., see Brennan

Wells, M. J. and Peay, A., Interdisciplinary Treatment of Musculoskeletal Pain: The EMPOWER Program, 3(1):54-63 


\section{SUBJECT INDEX}

Analgesia, 3(2):53-59

Analgesic ladder, 3(2):7-26

Ankles, 3(3):57-68

Assessment, 3(1):20-47

Back pain, 3(2):44-52, 3(4):1-13

Biomechanics, 3(3):31-43, 3(3):44-56

Bone metastasis, 3(2):1-6

Braces, 3(3): 12-20

Bracing, 3(3):1-11, 3(3):21-30,

$3(3): 44-56,3(4): 50-60$

Brachial plexus, 3(2):35-43

CT, 3(2):35-43

Cancer pain, 3(2)53-59, 3(2):69-77

Case management, 3(1):76-82

Casting, 3(3):57-68

Cervical dysfunction, 3(4):80-85

Cervical forward bending, 3(4):80-85

Chronic disability syndrome 3(1):6-19

Chronic pain, 3(1):6-19, 3(1):20-47 $3(1): 48-53,3(1): 54-63,3(1): 64-70$, $3(1): 71-75,3(1): 83-87,3(1): 88-90$

Chronic pain magnification, $3(1): 6-19$

Chronic pain programs, 3(1):83-87, 3(1):88-90

Claim handler, 3(4):61-67

CNS dysfunction, 3(2):1-6

Communication, 3(3):ed.

Deconditioning, 3(2):1-6

Degenerative, 3(4):24-31

Diagnosis back pain, 3(4):1-13

Disability, 3(4):32-35

Disability determination factors,

$3(1): 6-19,3(1): 20-47,3(1): 64-70$,

$3(1): 71-75$

Disability evaluations, 3(1):64-70,

3(1):71-75

Durable medical equipment, 3(3):ed

Elastic, 3(3):12-20

EMPOWER, 3(1):54-63

Epidural disease, 3(2):44-52

External case management,

3(1):76-82

Facet cysts, 3(3):69-73

Functional, 3(4):50-60

Functional capacity, 3(1):6-19

Functional capacity evaluation,

$3(4): 61-67,3(4): 86-94$
Functional progression, 3(4):36-49

Functional rehabilitation, 3(4):36-49

Illness behavior, 3(1):20-47

Impairment, 3(1):6-19, 3(1):64-70, 3(1):71-75

Industrial rehabilitation, 3(4):86-94

Injured worker, $3(1): 83-87$,

$3(1): 88-90,3(4): 61-67$

Interdisciplinary treatment, 3(1):54-63

Internal case management, 3(1):76-82

Karnofsky score, 3(2):1-6

Lifestyle change, 3(4):1-13

Low back pain, 3(1)48-53, 3(3):1-11, $3(3): 12-20,3(3): 69-73,3(4): 14-22$, 3(4):32-35

Lumbar sacral function, 3(4):24-31

Lumbar supports, 3(3):12-20

Lumbosacral plexus, 3(2):35-43

MPI, 3(1):20-47

Malingering, 3(1):6-19

Medical devices, 3(3):ed.

Magnetic resonance imaging (MRI), 3(2):35-43, 3(3):69-73

Metastases, 3(2):35-43, 3(2):53-59

Metastases to bone, 3(2):27-34, $3(2): 78-84$

Modalities, 3(2):53-59

Multidisciplinary management, 3(1):48-53

Muscle stabilization, 3(4):36-49

Musculoskeletal pain, 3(1):54-63

Myelosuppression, 3(2):60-68

Neoplasm, 3(2):35-43

Neospinothalamic, 3(1):1-5

Neurophysiology, 3(1):1-5

Nociception, 3(1): 1-5

Nonopioid analgesics, 3(2):7-26

Oncology rehabilitation, 3(2):78-84

Opioids, 3(2):7-26

Orthoses, 3(3):1-11, 3(3):21-30, $3(3): 44-56$

Orthosis, 3(3):ed.

Orthotics, 3(2):53-59, 3(3):1$11,3(3): 31-43$

Osseous disease, 3(2):60-68

Osteopenia, 3(3):44-56

Osteoporosis, 3(3):44-56

Pain, 3(1):1-5, 3(3):1-5, 3(1):6-19 $3(2): 78-84,3(4) 68-79$
Pain behavior, 3(1):54-63

Pain center, $3(1): 48-53$

Pain gate, $3(1): 1-5$

Pathologic fracture management, 3(2):27-34

Peripheral neurologic disease, $3(2): 1-6$

Physiatric intervention, 3(2):53-59

Physiatric management, 3(2):60-68, 3(2):69-77

Physical impairments, 3(2):1-6

Physical therapy, 3(2):78-84

Progressive, 3(4):50-60

Prosthesis, 3(3):ed.

Psychological factors, 3(1):20-47

Radiotherapy, 3(2):69-77

Rehabilitation, 3(3):31-43,

$3(3): 44-56,3(4): 50-60,3(4) 68-79$

Rehabilitation counselor, 3(4):61-67

Rehabilitation nurse, 3(4):61-67

Residual functional capacity, 3(1):6-19

Resistance training, 3(4):32-35

Return to work, 3(4):61-67

Sacroiliac joint dysfunction, $3(3): 31-43$

Second opinion, 3(4): ed.

Shear stressors, 3(4):24-31

Sickness impact profile, 3(1):20-47

Social security, 3(1):64-70, 3(1):71-75

Spinal metastases, 3(2):69-77

Spinal orthotics, 3(3):1-11

Sphincteric dysfunction, 3(2):44-52

Spinal supports, 3(3):21-30

Spondylolisthesis, 3(4):14-22, 3(4):24-31

Sports injuries, 3(3):57-68

Sprains, 3(3):57-68

Stabilization, 3(4):50-60

Strain energy, 3(3):12-20

Strategies for back pain, 3(4):1-13

Supports, 3(3):21-30, 3(3):57-68

Taping, 3(3):57-68

Therapeutic dependence, 3(2):7-26

Thoracic segmental flexion, 3(4):80-85

Torso strengthening, 3(4):32-35

Work capacity evaluation, 3(4):86-94

Work hardening, 3(4):86-94

Workers compensation, 3(1):83-87, 3(1):88-90 